INPLASY

PROTOCOL

To cite: Zhang et al. Atrial fibrillation And the Severe of Obstructive Sleep Apnea (OSA): A Dose-Response Meta-Analysis. Inplasy protocol 2020120104. doi: 10.37766/inplasy2020.12.0104

Received: 20 December 2020

Published: 21 December 2020

Corresponding author: Zhang Dong

466374026@qq.com

Author Affiliation:

The Department of

Cardiovascular Diseases, Xijing Hospital, Fourth Military

Medical University

Support: NSFC.

Review Stage at time of this submission: Formal screening of search results against eligibility criteria.

Conflicts of interest: None.

\section{Atrial fibrillation And the Severe of Obstructive Sleep Apnea (OSA): A Dose-Response Meta-Analysis}

\author{
Zhang, D1; Yu, ZX2; Yi, F³.
}

Review question / Objective: Explore association between OSA and different subgroups of AF , and try to find the doseresponse relationship between AF and OSA's different severity.

Condition being studied: Obstructive Sleep Apnea (OSA) is a common multifactorial disorder affecting both children and adults characterized by recurrent upper airway obstruction during sleep which leads to intermittent hypoxia. As highlighted, all organs and systems suffered from OSA with cardiovascular consequences. However, OSA is a poorly undervdiagnosed condition with increasing prevalence, especially in men. Atrial fibrillation (AF) is the most common arrhythmia found in patients 5 affecting $1-2 \%$ of the population around the world 6 with increased cardiovascular mortality due to stroke and heart failure. AF has been proved to be with an increasing prevalence with advancing age7. We planned to not only explore association between OSA and different subgroups of AF in a conventional meta-analysis but the dose-response relationship between AF and OSA's different severity from the related studies in a dose-response metaanalysis.

INPLASY registration number: This protocol was registered with the International Platform of Registered Systematic Review and Meta-Analysis Protocols (INPLASY) on 21 December 2020 and was last updated on 21 December 2020 (registration number INPLASY2020120104).

\section{INTRODUCTION}

Review question / Objective: Explore association between OSA and different subgroups of AF , and try to find the dose- response relationship between $\mathrm{AF}$ and OSA's different severity.

Rationale: Numerous studies have shown that OSA is associated with cardiovascular dysfunction, but the results of studies on 
OSA associated with atrial fibrillation have been inconsistent.

Condition being studied: Obstructive Sleep Apnea (OSA) is a common multifactorial disorder affecting both children and adults characterized by recurrent upper airway obstruction during sleep which leads to intermittent hypoxia. As highlighted, all organs and systems suffered from OSA with cardiovascular consequences. However, OSA is a poorly underdiagnosed condition with increasing prevalence, especially in men. Atrial fibrillation (AF) is the most common arrhythmia found in patients5 affecting 1-2\% of the population around the world6 with increased cardiovascular mortality due to stroke and heart failure. AF has been proved to be with an increasing prevalence with advancing age7. We planned to not only explore association between OSA and different subgroups of AF in a conventional metaanalysis but the dose-response relationship between AF and OSA's different severity from the related studies in a dose-response meta-analysis.

\section{METHODS}

Participant or population: The researches included all had clear outcomes and declared odds ratio (OR), relative risk (RR) or hazard ratio (HR) and $95 \%$ confidence interval $(\mathrm{Cl})$ for the association between OSA and AF. There were no restrictions on gender and age. We excluded the research where the researchers used other indexes except $\mathrm{AHI}$ as indicators. As for the research type, case reports, fundamental researches and reviews were also excluded.

Intervention: With OSA.

\section{Comparator: Without OSA.}

Study designs to be included: Observational study.

Eligibility criteria: The researches included all had clear outcomes and declared odds ratio (OR), relative risk ( $R R$ ) or hazard ratio
(HR) and 95\% confidence interval (CI) for the association between OSA and AF.

Information sources: PubMed, Embase, Cochran Library and web of science databases.

Main outcome(s): Mortality.

Additional outcome(s): Recurrence rate.

Quality assessment / Risk of bias analysis: Two investigators finished the conventional meta-analyses with Cochrane review manager version 5.3 software's help to assess a specific outcome's risk. The risk of bias was applied according to the Cochrane guidelines including random sequence generation, allocation concealment, blinding of participants and personnel, blinding of outcome assessment, incomplete outcome data, selective reporting, and other sources.

Strategy of data synthesis: The evaluation of heterogeneity among studies was undergone with the use of $Q$ and 12 . We adopt a standard for $P$-value that $P$ value $<0.1$ means the results possessed statistical heterogeneity. 12 describes the extent of variation due to heterogeneity rather than chance. The lower 12 is, the less variation is. $12<25 \%$ was considered little heterogeneity, $25 \%<1250 \%$ showed there existed enough heterogeneity to select a random effects-model. While $12<50 \%$, a fixed-effect model was employed. For further research, we performed a doseresponse meta-analysis using the Stata version 16.0 software. We underwent the dose-response meta-analysis based on a two-step method. First, the correlation between the $\mathrm{AHI}$ and the risk of $\mathrm{AF}$ was evaluated with a spline model. In this spline model, we took $\mathrm{AHI}$ as an independent variable and the $O R / R R$ as a dependent variable. We selected a corresponding merge model to merge the risk value for each study calculated in the first step due to the heterogeneity.

Subgroup analysis: We underwent a subgroup analysis due to the AF types. Roughly, the included studies could be 
divided into three subgroups, new-onset under no obvious incentive, new-onset after surgical operations like coronary artery bypass grafting (CABG) and reference $A F$ after ablation treatment.

Sensibility analysis: Sensibility analysis will be applied if the heterogeneity is obvious.

Country(ies) involved: China.

Keywords: Atrial fibrillation; Obstructive Sleep Apnea; Cardiovascular diseases; systematic review and Metaanalysis.

Contributions of each author:

Author 1 - Zhang Dong.

Email: 466374026@qq.com

Author 2 - Yu Zhi Xiang.

Email: xuebojun51@163.com

Author 3 - Yi Fu.

Email: yi12fu56@hotmail.com 